\title{
Intramedullary spinal cord solitary fibrous tumor: A rare clinical entity
}

\author{
Virendra R. Desai ${ }^{1}$, Robert A. Scranton ${ }^{1}$ and David Baskin ${ }^{1,2^{*}}$ \\ ${ }^{1}$ Department of Neurosurgery, Houston Methodist Hospital, Houston, TX 77030, USA \\ ${ }^{2}$ Kenneth R. Peak Brain and Pituitary Tumor Treatment Center, Houston, USA
}

\begin{abstract}
Introduction: Solitary Fibrous Tumor (SFT) is a rare tumor that initially was thought to arise from visceral tissue. Later reports documented its occurrence in central nervous system tissue, with most located intracranially. Few reports have mentioned intraspinal SFTs, with the majority being extra-medullary. In this report, we describe a rare presentation of intramedullary SFT.

Case description: A 52-year-old female was referred for neurosurgical treatment after a medical workup of low back and left leg pain revealed an intramedullary, enhancing mass at T11 with associated syrinx formation. The patient was taken to the operating room, and a multi-level thoracic laminectomy and durotomy was performed to expose the mass. A meticulous resection was initiated but only partially completed because of dense adherence to the spinal cord and nerve roots, with some nervous tissue contained inside the tumor. The patient's symptoms improved postoperatively. Histopathology documented the presence of a solitary fibrous tumor.

Discussion: Only 14 previous cases of intramedullary SFT have been reported. Differentiating SFT from other pathologies is important for correct intraoperative and post-operative management and follow-up. Although most cases of SFT are benign, certain histological characteristics denote the tendency for aggressive conversion, such as increased mitotic figures, nuclear atypia, and an increased proliferation index. In this study, we propose an approach to post-operative follow-up of a patient presenting with intramedullary SFT with benign histological characteristics.
\end{abstract}

\section{Introduction}

Solitary Fibrous Tumors (SFT) are rare and typically arise from visceral pleura, first described in 1931 using the term "localized fibrous mesothelioma" [1]. This tumor can also occur in non-serosal sites including the spinal cord. It is now believed that SFT arises from mesenchymal tissue [2].

After Carneiro et al. [3] published a seminal report on SFTs in the central nervous system in 1996, a flurry of other cases emerged, with most being intracranial (77.3\%). Of the intraspinal SFTs, the majority are dural-based and extra-medullary, with a small number being subpial, intraparenchymal, or involving nerve rootlets with no dural connection $[1,4]$.

There have been only 14 reported cases of intramedullary SFTs [2,4-12]. These patients had various presenting symptoms with most experiencing gradually progressive weakness and paresthesias associated with myelopathy, but normal bowel and bladder function and normal perineal sensation. Three cases reported a definite surgical plane between tumor and spinal cord, five reported no definite plane, and the six remaining case reports did not address this issue.

We report a patient who presented with an L5 radiculopathy, but after spinal imaging was found to have an intramedullary thoracic spinal mass in addition to a herniated lumbar disc. Interestingly, after resection of the thoracic mass, the patient experienced complete resolution of her L5 radicular syndrome.

\section{Case report}

\section{History and physical exam}

The patient is a 52 year-old female who presented with lower back and left leg pain. She reported pain in the lower back that was sharp in nature that radiated caudally along the posterior and lateral aspect of her thigh to the anterior surface of the leg and dorsum of the foot. The pattern was consistent with an L5 radicular syndrome. Examination revealed weakness in the left tibialis anterior and extensor hallucislongus without a sensory deficit. Deep tendon reflexes were diffusely depressed in her legs. The straight leg raising test was positive at 55 degrees on the left.

\section{Radiographic imaging}

A lumbar MRI scan with and without contrast demonstrated a left L4-5 disk herniation impinging on the left L5 nerve root as well as a large, enhancing mass at T11 involving nearly the entire thoracic cord. A thoracic MRI with and without contrast was subsequently performed showing an intraspinal lobulated mass with wellcircumscribed margins measuring 2 centimeters $(\mathrm{cm})$ cranio-caudal

Correspondence to: David S. Baskin, M.D., FACS, FAANS, Distinguished Chair Kenneth R. Peak Brain and Pituitary Tumor Treatment Center, Houston, USA; Tel: (713) 441-3800; Fax: (713) 793-1001; E-mail: dbaskin@houstonmethodist.org

Key words: central nervous system tumor,intramedullary tumor, spinal cord tumor, solitary fibrous tumor

Received: July 10, 2015; Accepted: August 17, 2015; Published: August 20, 2015 
(CC) $\times 1.3 \mathrm{~cm}$ transverse (TR) x $1.5 \mathrm{~cm}$ anteroposterior (AP) (Figure 1). The mass was iso-intense on $\mathrm{T} 1$ pre-contrast, hypo-intense on $\mathrm{T} 2$, and homogeneously enhancing with gadolinium. It displaced and compressed the spinal cord, causing increased T2 central cord signal intensity and syrinx formation superiorly to the bottom of the T9 vertebral level. The initial differential diagnosis included schwannoma, meningioma, and less likely Hemangiopericytoma(HPC) based upon the imaging characteristics.

\section{Operative management}

The patient was taken to the operating room for elective resection of the thoracic mass utilizing intraoperative monitoring of Somatosensory Evoked Potentials (SSEPs) and Motor Evoked Potentials (MEPs) to prevent spinal cord injury. A T11 laminectomy with partial T10 and T12 laminectomies were performed, exposing the dura overlying the mass (Figure $2 \mathrm{~A}$ ). The dura was then opened and a very large mass was seen occupying the majority of the spinal cord (Figure 2B). The arachnoid was dissected until the mass was exposed. No neural elements were seen overlaying the mass. Grossly the mass appeared to be a tan to yellow color and felt firm and rubbery. A portion of the mass was excised and sent to pathology for both frozen and permanent sections, with the frozen section showing fibrous tissue without neoplasm. A Cavitron Ultrasonic Surgical Aspirator (CUSA) (Integral Life Sciences Corporation, Plainsboro, New Jersey, USA) was used to debulk about half the tumor. At this point an effort to dissect

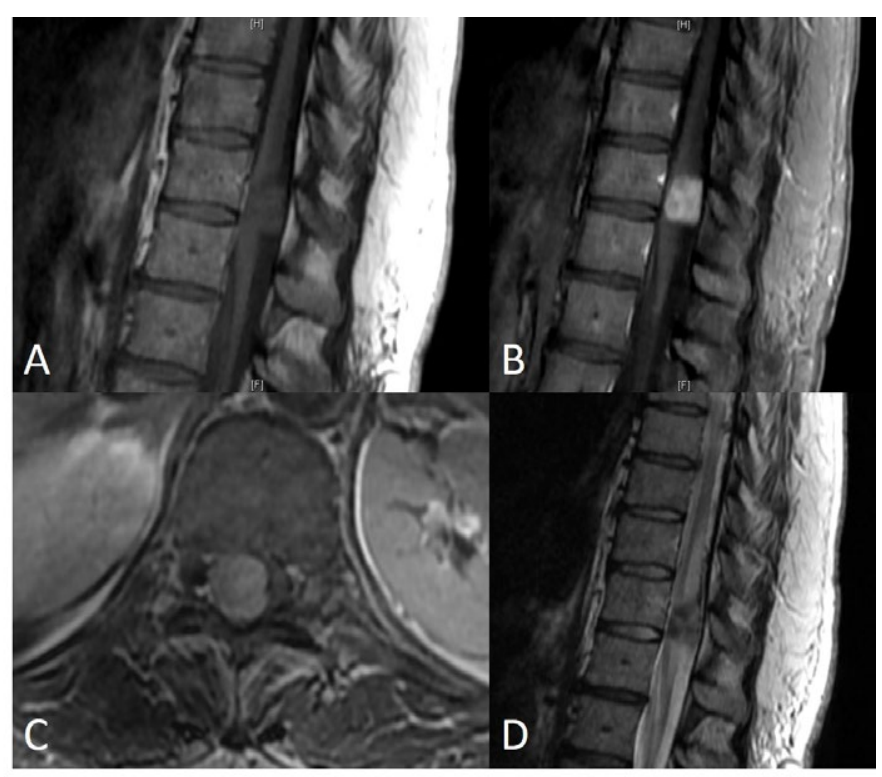

Figure 1. Preoperative MRI thoracic spine Sagittal T1 precontrast (A), postcontrast (B), axial $\mathrm{T} 1$ post contrast (C), and sagittal T2 (D).

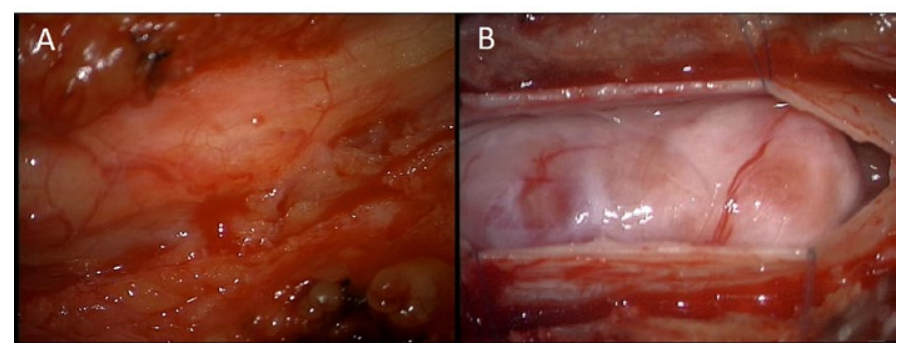

Figure 2. Intraoperative photographs showing epidural appearance (A) and intradural appearance (B). the spinal cord and nerve roots off the tumor was attempted, but aborted after dense adhesions to nerve roots and the spinal cord were found to be present. The nerve roots were not only adherent to the mass but appeared to go into and exit the mass. During the dissection and debulking there were abnormal transient changes in the SSEPs and MEPs, suggesting that continued dissection would likely lead to permanent neurologic deficits. Given the densely adherent nature of the tumor to the underlying neural structures and the frozen section analysis returning as only fibrous tissue, a decision was made to terminate the operation rather than risk producing new neurological deficits.

\section{Postoperative course}

Post-operative examination demonstrated the patient's strength to be $5 / 5$ in both lower extremities except for $4+/ 5$ strength in the right iliopsoas, although her exam was limited by pain. Her sensation to light touch and pinprick were intact. On postoperative day 1 her strength improved to $5 / 5$ in all muscle groups. During the remainder of the hospital course, the patient continued to do well and was discharged home on the fourth postoperative day.

The patient was seen in follow-up 2 weeks and 1 month postoperative. A contrasted thoracic MRI 1 month post-operative showed partial resection of the mass. The previously identified increased T2 cord intensity had decreased significantly with near complete resolution of the associated syrinx.

\section{Pathology}

The gross specimen sent to pathology contained dense fibrocollagenous tissue that appeared tannish-yellow in color. Microscopic examination showed dense fibrocollagenous tissue with granulation tissue. The CUSA aspirate showed spindle cells arranged in fascicles with eosinophilic cytoplasm and intervening collagen, consistent with solitary fibrous tumor (Figure 3). Mitotic figures were not easily identified. Immunohistochemistry was positive for CD34, Bcl-2 and CD99, and negative for pancytokeratin and Epithelial Membrane Antigen (EMA) (Figure 4). S-100 and neurofilament were negative in spindle cells, but positive in entrapped peripheral nerve axons. Smooth Muscle Actin (SMA) was non-contributory, while CD68 highlighted scattered macrophages and CD45 highlighted scattered lymphocytes. Ki-67 (MIB-1) showed a proliferation index up to $2 \%$ in the spindled areas. This was all consistent with the diagnosis of solitary fibrous tumor.

\section{Discussion}

SFTs are difficult to diagnose preoperatively solely on the imaging characteristics. Computed tomography can show heterogeneous isodense or hyperdense masses; the MRI appearance is isointense on $\mathrm{T} 1$ weighted images and hypointense on T2 weighted images. Contrast

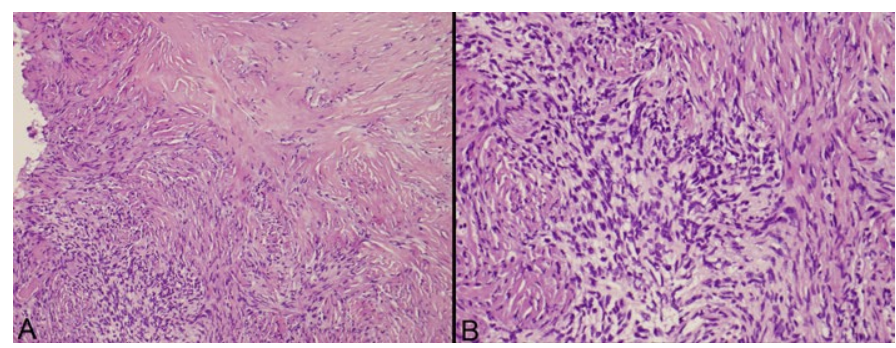

Figure 3. Photomicrographs of the tumor with hematoxylin and eosin staining at 10x and 20x magnification. 


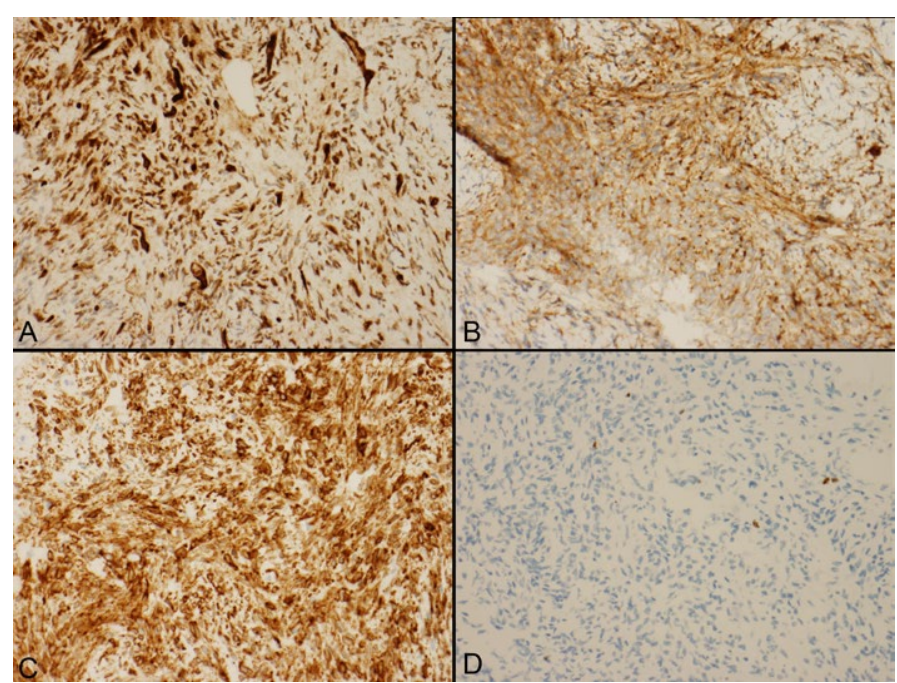

Figure 4. Photomicrographs of the tumor stained and strongly positive for CD34 (A), CD99 (B), Bcl-2 (C), and MIB-1 (D) of 2\% focally.

enhancement on both CT and MRI can be seen and is variable, being described as heterogeneous or homogeneous. Some authors state that the hypointense T2 signal is the best clue of SFT pathology. Additionally, a "ying-yang" sign ("patchy black and white" pattern with areas of hyperintensity and hypointensity on T2 sequences) may suggest SFT. This sign occurs because of staghorn vascularity and variations in cellularity $[1,8]$.

Grossly, almost all SFTs are well-circumscribed, smooth surfaced, and partially or completely (pseudo)encapsulated. Grossly the tumors are described as firm, mostly solid but occasionally with cystic components, with a gray-white to tan, whorled cut surface [1].

SFTs can be histologically similar to hemangiopericytoma, schwannoma, fibrous meningioma, fibroma, gliofibroma, and ependymoma [8]. Immunohistochemistry is vital to diagnosis of SFTs, with vimentin, $\mathrm{CD} 34, \mathrm{Bcl} 2$, and $\mathrm{CD} 99$ being the most consistently positive markers. CD34+ fibroblasts are normally found in dura, subpial interstice, perineurium and endoneurium of normal nerves and intraparenchymal perivascular connective tissue, and thus SFTs can arise from any of these locations [1]. Meningioma, HPC and SFT can stain positive for CD34; however meningioma and HPC will typically be positive for EMA while SFT will not [4]. Correct differentiation between SFT and hemangiopericytoma is important for postoperative management, as the latter behaves more aggressively and requires chemotherapy and radiation [8].

Solitary fibrous tumors are usually benign, but malignant cases have been reported [1,13-17]. Malignancy or high risk for recurrence is associated with: hypercellularity, marked nuclear atypia, high mitotic activity, necrosis, high Ki-67 proliferation index and sub-total resection $[1,4,18]$. Other signs of secondary malignant transformation are diminished CD34 expression and positive p53 immunoreactivity [18]. However, Munoz et al. [18] believe the histologic findings of SFT are unreliable for predicting behavior, as they presented a case of malignant SFT with multiple metastases that had benign histologic findings (Munoz, 2008). Authors note that the mean follow-up was only 40 months, and thus, the true long-term course of SFT is unknown $[1,4]$. Ki67/MIB1-LI proliferation index can help determine prognosis as $>5 \%$ is associated with higher rate of recurrence [1].

The relatively few number of spinal SFT cases means there is no clear recommendation on long-term follow-up. Bisceglia et al. [1] recommend biannual MRI scans for patients who had sub-total resection, atypical pathologic features, or high Ki67/MIB1-LI index $(>5 \%)$. If the patient bore a histologically typical SFT with gross total resection and low proliferative index, the authors advocated following the patient with annual MRI for 5 years, then MRI every 5 years thereafter [1]. We believe this is a reasonable approach in our patient, given that the mass did not have features suggestive of malignancy. The pathology showed a maximum Ki-67 of $2 \%$, no evidence of hypercellularity, no definite mitotic figures, no evidence of nuclear atypia and positive CD34 staining. If however the mass were to enlarge, or the patient develops new symptoms that could be related to the mass, a repeat surgical excision may be indicated.

\section{Acknowledgement}

This work was supported by the Donna and Kenneth R. Peak Foundation, The Kenneth R. Peak Brain and Pituitary Center at Houston Methodist Hospital, The Taub Foundation, The Blanche Green Estate Fund of the Pauline Sterne Wolff Memorial Foundation, The John S. Dunn Foundation, The Methodist Hospital Foundation, The Veralan Foundation, and The American Brain Tumor Association. We are particularly grateful to our patients and their families who have been impacted by the devastating effects of brain and spinal tumors and other central nervous system disease.

\section{References}

1. Bisceglia M, Galliani C, Giannatempo G, Lauriola W, Bianco M, et al. (2011) Solitary fibrous tumor of the central nervous system: a 15-year literature survey of 220 cases (August 1996-July 2011). Adv Anat Pathol 18: 356-392. [Crossref]

2. Alston SR, Francel PC, Jane JA Jr. (1997) Solitary fibrous tumor of the spinal cord. Am J Surg Pathol 21: 477-483. [Crossref]

3. Carneiro SS, Scheithauer BW, Nascimento AG, Hirose T, Davis DH (1996) Solitary fibrous tumor of the meninges: a lesion distinct from fibrous meningioma. A clinicopathologic and immunohistochemical study. Am J Clin Pathol 106: 217-224. [Crossref]

4. Tihan T, Viglione M, Rosenblum MK, Olivi A, Burger PC (2003) Solitary fibrous tumors in the central nervous system. A clinicopathologic review of 18 cases and comparison to meningeal hemangiopericytomas. Arch Pathol Lab Med 127: 432-439. [Crossref]

5. Kuchelmeister K, Scheuerle A, Bohle RM, Kretschmer T, Richter HP, et al. (1997) Solitary fibrous tumor: a new differential diagnosis in surgical neuropathology - report of two cases. Clinical Neuropathology 16: 270.

6. Mordani JP, Haq IU, Singh J (2000) Solitary fibrous tumour of the spinal cord Neuroradiology 42: 679-681.

7. Bohinski RJ, Mendel E, Aldape KD, Rhines LD (2004) Intramedullary and extramedullary solitary fibrous tumor of the cervical spine. Case report and review of the literature. J Neurosurg 100: 358-363. [Crossref]

8. Kawamura M, Izawa K, Hosono N, Hirano H (2004) Solitary fibrous tumor of the spinal cord: case report and review of the literature. Neurosurgery 55: 433. [Crossref]

9. Pizzolitto S, Falconieri G, Demaglio G (2004) Solitary fibrous tumor of the spinal cord: a clinicopathologic study of two cases. Ann Diagn Pathol 8: 268-275. [Crossref]

10. Jallo GI, Roonprapunt C, Kothbauer K, Freed D, Allen J, et al. (2005) Spinal solitary fibrous tumors: a series of four patients: case report. Neurosurgery 57: E195. [Crossref]

11. Ishii K, Nakamura M, Matsumoto M (2009) Intramedullary solitary fibrous tumor of the spinal cord. Journal of Orthopaedic Science 14: 450-454

12. Ciappetta P, D'Urso PI, Cimmino A, Ingravallo G, Rossi R, et al. (2010) Intramedullary solitary fibrous tumor of dorsal spinal cord. Neuropathology 30: 273-278. [Crossref]

13. Brucher JM, Hizawa K, Wechsler W (1970) Ultrastructure d'un fibrome intracerebralet possibilite de transformation maligne de ce type tumoral. Revue Neurologique 123: 443-445.

14. Burrig KF, Kastendieck H (1984) Ultrastructural observations on the histogenesis of 
localized fibrous tumors of the pleura (benign mesothelioma). Virchows Archiv [A] 403: 413-424.

15. Uzoaru I, Chou P, Reyes-Mugica M, (1984) Malignant solitary fibrous tumor of the pleura. Pediatric Pathology 14: 11-18.

16. Moran CA, Suster S, Koss MN (1992) The spectrum of histologic growth patterns in benign and malignant fibrous tumors of the pleura. Semin Diagn Pathol 9: 169-180. [Crossref]
17. Hanau CA, Miettinen M (1995) Solitary fibrous tumor: histological and immunohistochemical spectrum of benign and malignant variants presenting at different sites. Hum Pathol 26: 440-449. [Crossref]

18. Muñoz E, Prat A, Adamo B, Peralta S, Ramón y Cajal S, et al. (2008) A rare case of malignant solitary fibrous tumor of the spinal cord. Spine 33: E397-E399. [Crossref]

Copyright: (C2015 Desai VR. This is an open-access article distributed under the terms of the Creative Commons Attribution License, which permits unrestricted use, distribution, and reproduction in any medium, provided the original author and source are credited. 